\title{
Avaliação do pH e da condutividade elétrica do substrato sob níveis de fertirrigação e métodos de extração da solução ${ }^{(1)}$
}

\author{
POLIANA ROCHA D'ALMEIDA MOTA(2), ROBERTO LYRA VILLAS BÔAS(3), FERNANDA LUDWIG(3), \\ DIRCEU MAXIMINO FERNANDES ${ }^{(3)}$, MARCOS VINICIUS FOLEGATTI(4)
}

\begin{abstract}
RESUMO
O presente trabalho foi desenvolvido com o objetivo de avaliar o valor de $\mathrm{pH}$ e a condutividade elétrica (CE) da solução em equilíbrio com substrato sob níveis de fertirrigação e métodos de extração da solução. O estudo foi realizado em cultivo protegido no Departamento de Recursos Naturais/Ciência do Solo, da FCA/Unesp, de fevereiro a março de 2006. Adotouse o delineamento experimental em blocos casualizados utilizando o esquema fatorial 5 x 2 ( 5 níveis de CE e 2 métodos de extração) com cinco repetições. A CE das soluções aplicadas foi de 0,$5 ; 2,0 ; 3,5 ; 5,0$ e 6,5 dS m-1, e os métodos foram extrator de solução e "pourthru". O experimento foi conduzido em vaso plástico com volume de 1,3 L, sendo o substrato constituído da mistura de $30 \%$ de terra de subsuperfície e $70 \%$ casca de pinus fina. As quantificações da CE e do $\mathrm{pH}$ foram feitas aos 9, 16, 23, 37, 44 e 51 dias após o início das aplicações da fertirrigação. Os valores de pH e CE foram superiores quando a solução foi extraída pelo método "pourthru", devido ao local de extração e à característica física do substrato. Obteve-se coeficiente de correlação entre os dois métodos de $0,96^{* *}$ para a $\mathrm{CE}$ e de $0,87^{* *}$ para o $\mathrm{pH}$. Conclui-se que o uso do extrator de solução e o "pourthru" podem ser utilizados para avaliação do $\mathrm{pH}$ e da CE dos substratos, viabilizando o manejo da fertirrigação.
\end{abstract}

Palavras-chave: extrator de solução, "pourthru", manejo de nutrientes.

\section{ABSTRACT \\ pH and electrical conductivity of substrate submitted to the fertigation with different concentrations and extraction method}

\begin{abstract}
The present work was developed with the objective of evaluate the $\mathrm{pH}$ and electrical conductivity (EC) of substrate submitted to the fertigation with different concentrations and extraction method. The study was carried out in a greenhouse at Department of Natural Resources/Science of Soil, of the FCA/UNESP, of February to March of 2006. The experimental design were randomized blocks utilizing the plan factorial $5 \times 2$ ( 5 levels of electrical conductivity and 2 extraction method) with five repetitions. The levels of $\mathrm{CE}$ of the solution applied were: $0.5 ; 2.0 ; 3.5 ; 5.0$ and $6.5 \mathrm{dS} \mathrm{m}-1$, and the approaches: extractor of solution and pourthru. The experiment was conducted in potted with volume of $1.3 \mathrm{~L}$, being the substrate constituted of the mixture of $30 \%$ of land of subsurface and $70 \%$ fine pine bark. The quantification of the EC and of the $\mathrm{pH}$ was carried out to the $9,16,23,37,44$ and 51 days after the beginning of the application. The $\mathrm{pH}$ and $\mathrm{EC}$ were higher when the solution was extracted by the method pourthru due to the local extraction and the substrate physical characteristic. The $\mathrm{pH}$ and $\mathrm{EC}$ were higher when the solution was extracted by the pourthru due to the local extraction and the substrate physical characteristic. Was obtained coefficient of correlation between the two method of $0.96^{* *}$ to $\mathrm{CE}$ and $0.87^{* *}$ to $\mathrm{pH}$. The conclusion was the use of the extractor of solution and the pourthru they can be utilized for evaluation of the $\mathrm{pH}$ and of the EC of the substrate, making feasible the management of the fertigation.
\end{abstract}

Keywords: extractor of solution, pourthru, nutrient management.

\section{INTRODUÇÃO}

A nutrição da planta é fundamental para a adequada produção, especialmente na floricultura, podendo influenciar o tempo de cultivo, a qualidade e o custo de produção. A utilização de substrato em recipientes permite ao produtor maior controle do $\mathrm{pH}$ e manejo dos nutrientes. Contudo, esse rigoroso controle requer intensivo monitoramento para manter os parâmetros nutricionais em níveis adequados ao desenvolvimento das culturas. Um protocolo de teste de substrato simples, rápido e de qualidade é necessário para fornecer ao produtor a decisão do manejo da fertilização de forma prática (CAVINS, 2002).
O suprimento eficiente de nutrientes é imprescindível na produção de flores, pois resulta na sua disponibilidade para as plantas. O controle da fertirrigação, visando ao manejo adequado, pode ser feito pela quantificação do $\mathrm{pH}$ e da condutividade elétrica (CE) durante o ciclo de cultivo. BURGUEÑO (1996) sugere o monitoramento da salinidade, ou seja, da concentração iônica por intermédio de medidas sistemáticas da condutividade da solução do solo e até mesmo a tomada de decisão quanto ao momento e quantidade de fertilizantes a serem aplicados via água de irrigação. Este manejo, realizado como rotina, pode prevenir muito dos problemas relacionados à nutrição.

Ainda existem poucas informações e muitas

\footnotetext{
(1) Recebido em 29/10/2009 e aceito para publicação em 27/05/2011

*Os autores agradecem à Fundação de Amparo à Pesquisa do Estado de São Paulo (Fapesp), pelo auxílio à pesquisa (Processo No 05/60680-9); ao Conselho Nacional de Desenvolvimento Científico e Tecnológico (CNPq), pela concessão de bolsa de estudo; ao Ministério da Ciência e Tecnologia (MCT), ao CNPq e à Fapesp, pelo apoio financeiro através do Instituto Nacional de Ciência e Tecnologia - Engenharia da Irrigação (INCT-EI).

(2) Pós-Doutoranda, Instituto Nacional de Ciência e Tecnologia - Engenharia da Irrigação, ESALQ/USP. Av. Pádua Dias, 11, Piracicaba, SP, CEP 13418900, fone (19) 3447-8562, e-mail: polimota@yahoo.com.br. Bolsista do CNPq. Autor para correspondência;

${ }^{(3)}$ Departamento de Recursos Naturais/Ciência do Solo, FCA/Unesp, Botucatu, SP, CEP 18610-307, email: rlvboas@fca.unesp.br, fludwig@fca.unesp. br,dmfernandes@fca.unesp.br;

${ }^{(4)}$ ESALQ/USP, email: mvfolega@esalq.usp.br.
} 
controvérsias quanto aos métodos analíticos, faltando padronização dos procedimentos em laboratórios, para tornar possível a comparação dos resultados e, principalmente, a definição das faixas críticas para sua interpretação e recomendação de correção e adubação (SANTOS, 2005). O método de diluição de 1:5 (uma parte de substrato para 5 de água) foi estabelecido pelo Ministério da Agricultura, Pecuária e Abastecimento como padrão para caracterização inicial de substratos para plantas, segundo a instrução normativa n.17 de 21 de maio de 2007 (BRASIL, 2007). O extrato de saturação (RICHARDS, 1954) é considerado método padrão, enquanto a diluição de 1:2 é utilizada rotineiramente por produtores. Por apresentarem metodologias destrutivas, muitas vezes torna-se difícil e onerosa a rotina de análise, além da possibilidade de solubilização de alguns sais, devido ao excesso de diluição.

Métodos não destrutivos e que se utilizam do substrato na condição de campo são alternativas para o manejo da sua concentração de sais. $\mathrm{O}$ extrator de solução e o "pourthru" são métodos que atendem a essa demanda. Essas metodologias vêm sendo adotadas por produtores de flores, porém sem o devido conhecimento da relação entre elas, pois cada metodologia apresenta suas particularidades.

SILVA et al. (1999) citam que o uso do extrator de solução provido de cápsulas porosas, em umidades próximas à capacidade máxima de retenção de água, é de fácil execução, e a solução corresponde à umidade equivalente ao momento em que a solução é absorvida pela planta. Assim, os solutos dissolvidos são os mesmos que a planta estaria absorvendo, além de possibilitar uma amostragem sistemática, pontual e não destrutiva, sendo ainda a aferição da CE praticamente instantânea.

O método do "pourthru" se baseia no deslocamento de um volume de solução, adicionado na parte superior do substrato, com o objetivo de obter amostras de nutrientes através da solução lixiviada (CAVINS, 2002). É um método simples, rápido e que não necessita de equipamentos especializados (WRIGHT, 1986), não exige remoção do substrato e não há distúrbio para a raiz, no momento da extração (YAGER et al., 1983).

Devido à escassez de pesquisas relacionando metodologias, o presente trabalho foi desenvolvido com o objetivo de avaliar o valor de $\mathrm{pH}$ e a $\mathrm{CE}$ de substrato sob níveis de fertirrigação e métodos de extração da solução.

\section{MATERIAL E MÉTODOS}

O estudo foi desenvolvido em cultivo protegido no Departamento de Recursos Naturais/Ciência do Solo, da Faculdade de Ciências Agronômicas da Unesp, situado no município de Botucatu, Estado de São Paulo, de fevereiro a março de 2006. A temperatura média no interior da casa de vegetação foi de $25,5^{\circ} \mathrm{C}$ e $68,1 \%$ a umidade relativa do ar.

Adotou-se o delineamento experimental em blocos casualizados, utilizando o esquema fatorial 5 × 2 ( 5 níveis de condutividade elétrica e 2 métodos de extração) com cinco repetições. Os níveis de $\mathrm{CE}$ da solução aplicada foram 0,$5 ; 2,0 ; 3,5 ; 5,0$ e $6,5 \mathrm{dS} \mathrm{m}^{-1}$ e os métodos do extrator de solução e "pourthru".

O experimento foi conduzido em vaso plástico com volume de 1,3 L ( $\mathrm{n}^{\mathrm{o}} 15$ ), com dimensões de $12,2 \mathrm{~cm}$ de altura, $14,8 \mathrm{~cm}$ de base superior e $9,8 \mathrm{~cm}$ de base inferior. O substrato consistiu numa mistura de $30 \%$ de terra de subsuperfície e $70 \%$ casca de pinus fina. Cada vaso constituiu uma parcela.

O substrato apresentou as seguintes características físicas: densidade úmida de $732 \mathrm{~kg} \mathrm{~m}^{-1}$ e seca de 691 $\mathrm{kg} \mathrm{m}{ }^{-1}$, espaço de aeração de $6,47 \%$, água facilmente disponível de $18,7 \%$, distribuição do tamanho de partículas de $6,79 \%$ (partículas $>4 \mathrm{~mm}$ ), 14,16\% (4 a $2 \mathrm{~mm}$ ), 13,29\% (2 a $1 \mathrm{~mm}), 21,71 \%$ (1 a $0,5 \mathrm{~mm}), 25,01 \%(0,5$ a $0,25 \mathrm{~mm})$ e $19,04 \%(<0,25 \mathrm{~mm})$; características químicas: $\mathrm{CE}_{1: 5}$ de $0,56 \mathrm{dS} \mathrm{m}^{-1}$ e pH$_{1.5}$ de 6,62 .

A fertirrigação foi realizada manualmente de modo que cada vaso recebesse as quantidades preestabelecidas de nutrientes e o mesmo volume. As concentrações dos nutrientes foram baseadas em MOTA (2007), e as soluções aplicadas na quantidade de $100 \mathrm{~mL}^{\text {vaso }}{ }^{-1}$, duas vezes por semana, de modo que não houvesse lixiviação.

$\mathrm{O}$ monitoramento da $\mathrm{CE}$ foi realizado ajustando a quantidade de sais aplicados para a manutenção dos valores previstos para os tratamentos. As soluções foram manejadas de forma a serem mantidas no intervalo de tolerância de $20 \%$ acima ou abaixo da CE preestabelecida de acordo com metodologia proposta por MOTA (2007).

As avaliações da $\mathrm{CE}$ e do $\mathrm{pH}$ foram realizadas aos 9, $16,23,37,44$ e 51 dias após o início das aplicações (DAA) da fertirrigação. As extrações com os dois métodos eram realizadas sempre no mesmo dia e mesmo vaso, com o substrato na capacidade máxima de retenção de água.

Para o método do extrator de solução, seguiu-se a metodologia proposta por MOTA (2004). Foram instalados um extrator por vaso, confeccionado com tubo de PVC, e uma cápsula porosa de cerâmica em sua extremidade, na profundidade de $9,5 \mathrm{~cm}$. Para proporcionar o fluxo da solução do substrato para o extrator, retirava-se o ar de dentro dos extratores criando uma sucção interna de aproximadamente $-70 \mathrm{kPa}$ e se coletava a solução com o uso de uma seringa conectada a uma mangueira de silicone. A extração era realizada uma hora após a fertirrigação.

Para o método "pourthru", seguiu-se a metodologia proposta por CAVINS et al. (2000). O método consistiu em saturar o substrato com a solução nutritiva aplicada na porção superior do vaso até o início da sua percolação. Estes vasos eram deixados em repouso pelo período de uma hora, quando então recebiam um coletor na sua parte inferior, e a adição de $75 \mathrm{ml}$ de água destilada na sua porção superior. A água destilada deslocava a solução da parte inferior do vaso, que era coletada e então analisada.

Após a coleta da solução pelos dois métodos, foram determinados os valores de $\mathrm{CE}$ e $\mathrm{pH}$. Para a determinação da CE, foi utilizado condutivímetro portátil da marca Digimed, modelo DM-3; e para o pH, pHmetro portátil da marca Gehaka, modelo PG 1400.

Os efeitos dos tratamentos foram submetidos à análise de regressão, tendo sido testados os modelos linear e quadrático e escolhidos com base na significância dos coeficientes de regressão a $1 \%(* *)$ e $5 \%(*)$ de probabilidade pelo teste $\mathrm{F}$ e no maior valor do coeficiente 
de determinação $\left(\mathrm{R}^{2}\right)$. Os dados foram correlacionados com o uso do programa SigmaStat 3.5.

\section{RESULTADOS E DISCUSSÃO}

Os valores médios da $\mathrm{CE}$ da solução do substrato diferiram significativamente em relação à metodologia (Figura 1). A solução extraída pelo método "pourthru" apresentou maior $\mathrm{CE}$ em relação àquela extraída pelo extrator, e essa diferença se deve principalmente à forma e ao local de extração em cada um deles.

Os menores níveis de CE quantificados pela metodologia do extrator podem ser justificados pela sua determinação pontual, já que os extratores eram posicionados a $9,5 \mathrm{~cm}$ da porção superior do vaso, limitando seu raio de absorção. Com a metodologia do "pourthru" ocorre o arraste dos sais de um volume maior do substrato, ampliando a faixa a ser determinada.

Porém esta resposta é dependente do sistema de irrigação utilizado, pois de acordo com CAVINS (2002), a irrigação na parte superior do substrato pode promover o deslocamento dos nutrientes por fluxo de massa para a parte inferior do vaso. Devido à natureza de deslocamento, o "pourthru" avalia somente a solução da porção inferior do recipiente (WRIGHT, 1986) e sendo essa a porção de maior concentração de sais, os valores de CE tendem a se elevar em comparação a métodos com outros pontos de coleta.

Durante o período de cultivo, gradientes de CE e pH podem se desenvolver ao longo do período do perfil vertical do substrato devido à diferença de aeração, conteúdo de água e evaporação da água para a superfície do substrato. Isso sugere que a posição do extrator no perfil do substrato pode afetar efetivamente os valores de $\mathrm{pH}, \mathrm{CE}$ e nutrientes na solução (JEONG, 2010).

Outro fator que pode ser considerado é a proporção de partículas finas no substrato $(19 \%$ de partículas $<0,25$ $\mathrm{mm})$, que tendem a se acomodar na porção inferior do vaso, devido a arranjo durante o preenchimento e movimentação dos vasos, além do arraste pela água de irrigação. De acordo com FERNANDES e CORÁ (2004), durante o período de cultivo, substratos com partículas bem distribuídas em muitas classes de tamanhos apresentam movimento das partículas menores nos espaços formados entre as partículas maiores, acomodando-se e reduzindo a porosidade total. Quanto menor o tamanho da partícula, maior a retenção de água e nutrientes, aumentando a $\mathrm{CE}$, quantificada pelo método "pourthru".

No entanto, CABRERA (1998), comparando os valores de $\mathrm{CE}$ extraídos pelo uso de cápsula porosa e do "pourthru", verificou que foram ligeiramente inferiores, porém não significativos, quando a solução foi extraída pelo "pourthru", indicando ser efeito da maior diluição. Esse autor, porém, realizou procedimentos diferenciados em relação ao presente trabalho, justificando os resultados discordantes. O autor obteve também elevada correlação entre os dois métodos e o extrato de saturação, sugerindo que os métodos não destrutivos são eficazes para indicar o estado nutricional na fase líquida de substratos em recipientes.

Ao longo do período de amostragem, ocorreu redução nos valores de $\mathrm{CE}$, mesmo não havendo planta para absorver os nutrientes adicionados (Figura 1). Possivelmente tenha ocorrido a interação do solo, que representava $30 \%$ da composição do substrato, com os íons, resultando na retenção destes mesmos íons. Essa pode ser uma característica importante para as plantas em que não há aplicação diária da fertirrigação, pois a retenção de sais nas partículas de solo que compõem o substrato resulta em fonte de reserva para suprir necessidades futuras das plantas.

Outra hipótese é de que, com a evaporação da solução, os sais tenderiam a se acumular na superfície do vaso. Com isso, a metodologia que utiliza o extrator não expressava a CE aplicada por ter a cápsula porosa um raio de alcance limitado (PIRES et al., 2007; ARTHUR, 2005), assim como o método do "pourthru" que tende a amostrar a porção inferior do vaso (CAVINS, 2002). Valores elevados de CE na porção superior do substrato resultam do acúmulo de sais devido à evaporação na superfície do substrato (HANDRECK e BLACK, 2002). Essa constatação fica ainda mais evidente no presente trabalho, já que não havia plantas que pudessem absorver os nutrientes ou reduzir a superfície de evaporação.

O fenômeno de acúmulo de sais na superfície do substrato é destacado por SONNEVELD (2000) e de forma mais evidente por ZHENG et al. (2004), em subirrigação para a cultura da gérbera.

Diferença significativa entre os valores de $\mathrm{pH}$ da solução do substrato extraída com os dois métodos foi observada aos 9, 23 e 37 DAA (Figura 2), superior para o "pourthru". LUDWIG et al. (2007) não registraram variação para os níveis de $\mathrm{pH}$ ao analisar o método do "pourthru" e a diluição do 1:2 (v/v).

Os valores médios de $\mathrm{pH}$ da solução do substrato obtida pelas duas metodologias apresentaram relação linear negativa em relação aos níveis de CE aplicados (Figura 2). De acordo com VAN IERSEL (1999), essa correlação negativa entre $\mathrm{CE}$ e $\mathrm{pH}$ é esperada, devido à acidez provocada pela adição dos fertilizantes.

A CE quantificada pelos dois métodos apresentou correlação linear positiva de $0,93 * *$ (Figura 3 ), validando os métodos para estimar a CE da solução do substrato em vaso. CABRERA (1998) registrou correlação positiva para a CE pelos mesmos métodos, além de correlação positiva desses com o extrato de saturação, método considerado padrão para determinação da CE. Correlações positivas altamente significativas para a CE foram destacadas também entre o extrator de solução e a diluição do 1:2 (MOTA et al., 2006), o "pourthru" e o teor total de sais solúveis (TTSS), que utiliza a diluição do 1:10 (SCHÄFER e SOUZA, 2005).

De forma semelhante, os valores de $\mathrm{pH}$ da solução do substrato extraída pelos dois métodos apresentaram correlação linear positiva de $0,87 * *$ (Figura 3 ). Correlação positiva altamente significativa foi registrada também por CABRERA (1998) ao comparar o extrator de cápsula porosa "pourthru" e o extrato de saturação, e por SCHÄFER e SOUZA (2005), ao comparar o "pourthru" e o método de diluição de 1:2,5.

Esses resultados permitem considerar os métodos avaliados adequados para quantificação, monitoramento e 
manejo da CE e pH em substrato. No entanto, é necessário o desenvolvimento de novas pesquisas com o objetivo de determinar valores padrão para as culturas.

Uma questão prática e importante a ser considerada quanto à escolha da metodologia utilizada é a determinação da característica física do substrato. Substratos com elevada porosidade e especialmente elevado espaço de aeração podem dificultar a utilização do extrator de solução, pois existe a necessidade de completa aderência da cápsula ao substrato para possibilitar a aplicação do vácuo para sucção da solução. No substrato utilizado, com $6,47 \%$ de espaço de aeração, não houve limitação ao uso do método. Para o método "pourthru", essa questão também é relevante, pois quanto menor o espaço de aeração e maior a retenção de água, a quantidade de água adicionada para promover o deslocamento e o tempo de estabilização podem sofrer alteração.

De acordo com CAVINS (2002), a elevada porosidade, boa drenagem, baixa densidade permitem ao "pourthru" ser considerado o método ideal para deslocamento da solução do substrato em recipientes. Para JEONG (2010), os dois métodos de extração fornecem amostras sem que haja algum tipo de perturbação no substrato, porém o extrator tem a vantagem de não implicar a substituição da solução do substrato com água e retirar uma pequena quantidade de amostra, reduzindo o impacto sobre o crescimento da planta.

\section{CONCLUSÃO}

Os métodos com uso do extrator de solução e o método do "pourthru" podem ser utilizados para avaliação do pH e da CE dos substratos em vaso, viabilizando o manejo da fertirrigação.

\section{REFERÊNCIAS}

ARTHUR, R. C. J. Determinação do raio de influência de extratores de solução do solo e de tensiômetros utilizando a técnica de tomografia computadorizada de raios gama. Piracicaba: Centro de Energia Nuclear na Agricultura, CENA, 2005. 51p. Dissertação (Mestrado em Energia Nuclear na Agricultura e no Ambiente).

BRASIL. Instrução Normativa n.17, de 21 de maio de 2007. Aprova os Métodos Analíticos Oficiais para Análise de Substratos e Condicionadores de Solos. Diário Oficial da União, Brasília, 24 maio. 2007. Seção 1, p.8.

BURGUEÑO, H. La fertirrigacion en cultivos hortícolas com acolchado plástico. Culiacan, 1996. v.1, 45p.

CABRERA, R. I. Monitoring chemical properties of container growing media with small soil solution samplers. Scientia Horticulturae, Amsterdam, v.75, n.1-2, p.113$119,1998$.

CAVINS, T. J. Adaptation of the pourthru nutrient extraction procedures to greenhouse crop production. Raleigh: Faculty of North Carolina State University, 2002. 148p. Tese (Doutorado).
CAVINS, T. J.; WHIPKER, B. E.; FONTENO, W. C.; HARDEN, B.; McCALL, I.; GIBSON J. L. Monitoring and managing $\mathrm{pH}$ and $\mathrm{EC}$ using the PourThru extraction method. Horticulture Information Leaflet, North Carolina, v.590 p.1-17, 2000.

FERNANDES, C.; CORÁ, J. E. Bulk density and relationship air/water of horticultural substrate. Scientia Agricola, Piracicaba, v.61, n.4, p.446-450, 2004.

HANDRECK, K.; BLACK, N. Salinity: a growing problem. In: Growing media for ornamental plants and turf. University of New South Wales Press., Sydney, Australia. 2002. 542 p.

JEONG, K Y. Establishment and stabilization of $\mathbf{p H}$ in container root substrate. Faculty of North Carolina State University, Raleigh: 2010. 95p. Tese (Doutorado).

LUDWIG, F.; FERNANDES, D. M.; MOTA, P. R. D.; VILLAS BOAS, R .L. Condutividade elétrica e $\mathrm{pH}$ do substrato em cultivares de gérbera de vaso avaliado com duas metodologias. In: CONGRESSO BRASILEIRO DE FLORICULTURA E PLANTAS ORNAMENTAIS, 16, Goiânia, 2007, Anais... Goiânia, 2007.

MOTA, P. R. D. Níveis de condutividade elétrica da solução do substrato em crisântemo de vaso, em ambiente protegido. Botucatu: Universidade Estadual Paulista, Faculdade de Ciências Agronômicas, 2004. 82p. Dissertação (Mestrado em Agronomia/Irrigação e Drenagem).

MOTA, P. R. D. Aplicação via fertirrigação de soluções com diferentes condutividades elétricas para produção de gérbera (Gerbera jamesonii L.) sob ambiente protegido. Botucatu: Universidade Estadual Paulista, Faculdade de Ciências Agronômicas, 2007. 149p. Tese (Doutorado em Agronomia/Irrigação e Drenagem).

MOTA, P. R. D.; VILLAS BOAS, R. L.; SOUSA, V. F. de. Concentração de sais na solução avaliada pela condutividade elétrica na zona radicular do crisântemo sob irrigação por gotejamento. Irriga, Botucatu, v.11, n.4, p.532-542, 2006.

PIRES, L. F.; ARTHUR, R. C. J.; BACCHI, O. O. S.; REICHARDT, K. Application of gamma-ray computed tomography to evaluate the radius of influence of soil solution extractors and tensiometers. Nuclear Instruments \& Methods in Physics Research. Section B. Beam Interactions with Materials and Atoms, England, v. 259, p. 969-974, 2007.

RICHARDS, L. A. Diagnostico y reabilitacion de suelos salinos y sodicos. Ed. México, 1954. 172p.

SANTOS, P. H. dos. Métodos de extração de micronutrientes em substratos para as plantas. Campinas: Instituto Agronômico de Campinas, 2005. 67p. Dissertação (Mestrado em Agricultura Tropical e 
Subtropical).

SCHÄFER, G. ; SOUZA, P. V. D. Método não destrutivo para monitoramento da reação e salinidade do substrato na produção de mudas cítricas. Laranja, Cordeirópolis, v.26, n.1, p.151-162, 2005.

SILVA, E. F. F.; MIRANDA, J. H.; COELHO, R. D. Determinação da salinidade do solo utilizando extratores de cápsulas porosas e soluções diluídas. (compact disc). In: CONGRESSO BRASILEIRO DE ENGENHARIA AGRÍCOLA, 28, Pelotas, 1999. Anais... Pelotas: SBEA, 1999.

SONNEVELD, C. Effects of salinity on substrate grown vegetables and ornamentals in greenhouse horticulture. Netherlands: Wageningen Universitiet, 2000. 149p. Tese (Doutorado).
VAN IERSEL, M. Fertilizer concentration affects growth and nutrient composition of subirrigation pansies. HortScience, Alexandria, v.34, n.4, p.660-663, 1999.

WRIGHT, R. D. The "pour-through" nutrient extraction procedure. HortScience, Alexandria, v.21, n.2, p.227-229, 1986.

YAGER, T. H.; WRIGHT, R. D.; DONOHUE, S. J. Comparison of "pour-through" and pine bark extract N, $\mathrm{P}, \mathrm{K}$ and $\mathrm{pH}$ levels. Journal of the American Society for Horticultural Science, Alexandria, v.108, n.1, p.112-114, 1983.

ZHENG, Y.; GRAHAM, T.; RICHARD, S.; DIXON, M. Potted gerbera production in a subirrigation system using low-concentration nutrient solutions. HortScience, Alexandria, v.39, n.6, p.1283-1286, 2004.
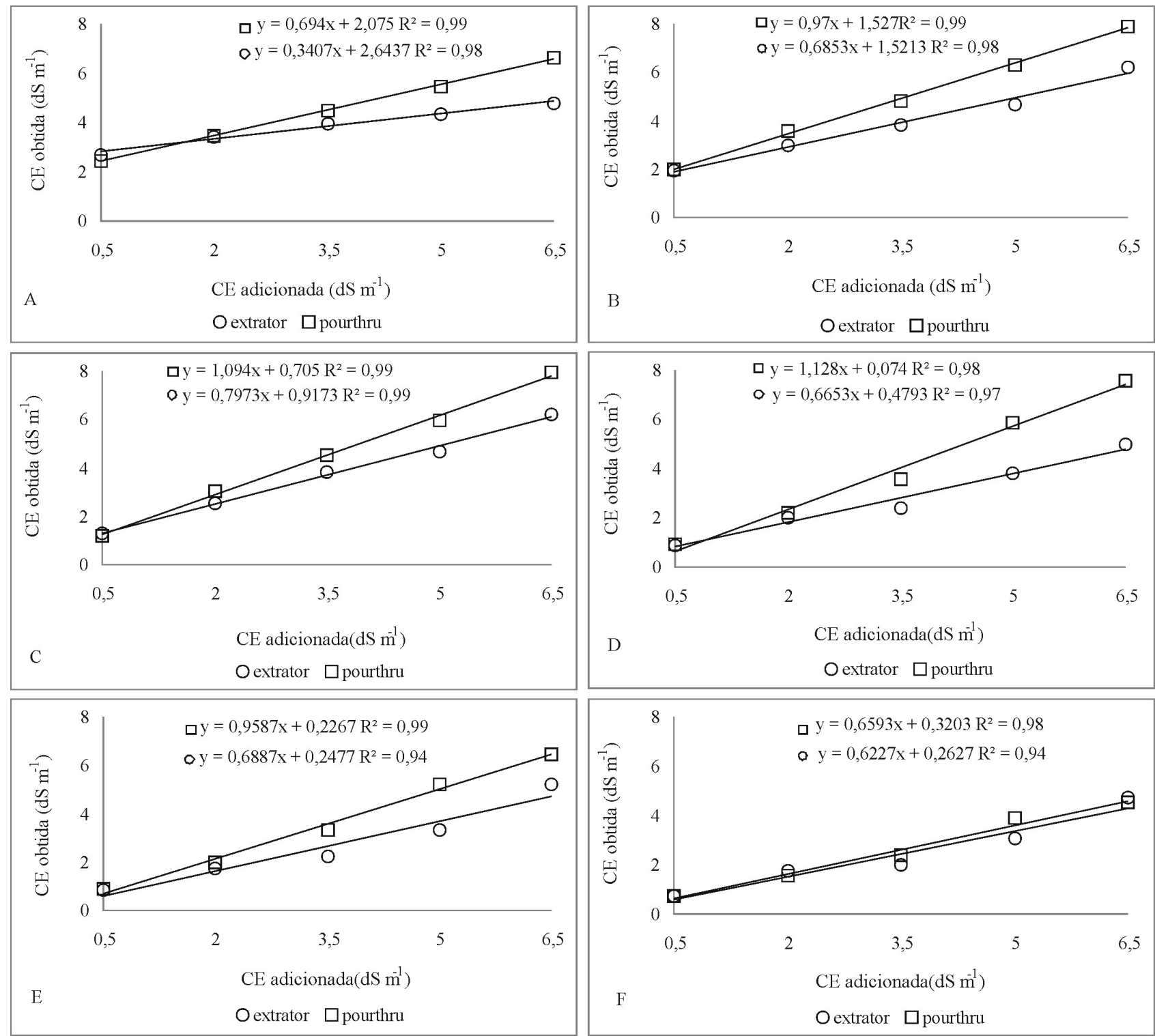

Figura 1. Condutividades elétricas obtidas pelas metodologias do extrator e "pourthru" em função das condutividades elétricas adicionadas aos 9 (A), 16 (B), 23 (C), 37 (D), 44 (E), 51 (F) dias após aplicação. Botucatu, UNESP. 2006.

Figure 1. Electrical conductivity obtained by the methods of solution extractor and pourthru as a function of electrical conductivity added, to 9 (A), 16 (B), 23 (C), 37 (D), 44 (E), 51 (F) days after application. Botucatu, UNESP. 2006. 

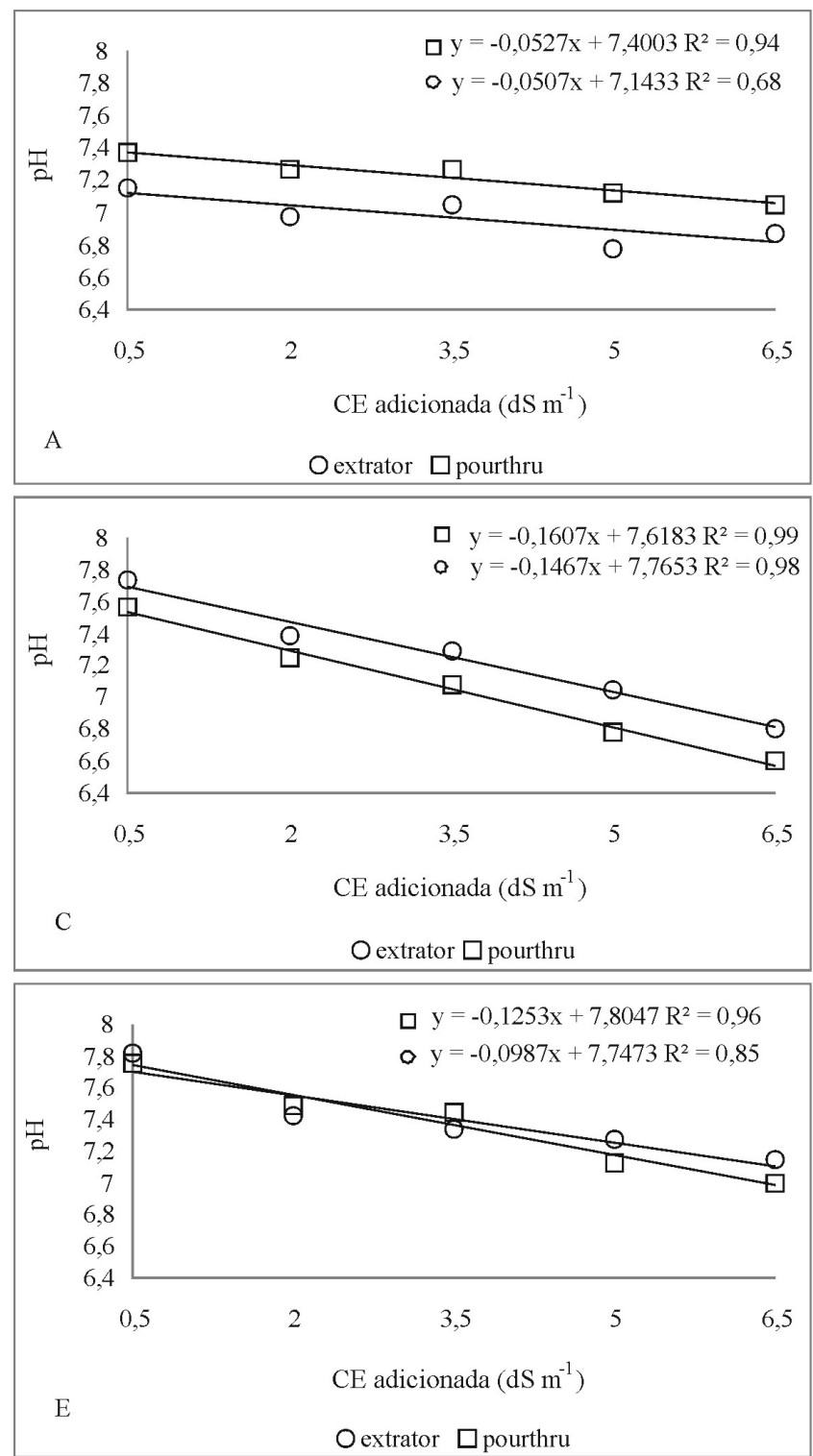
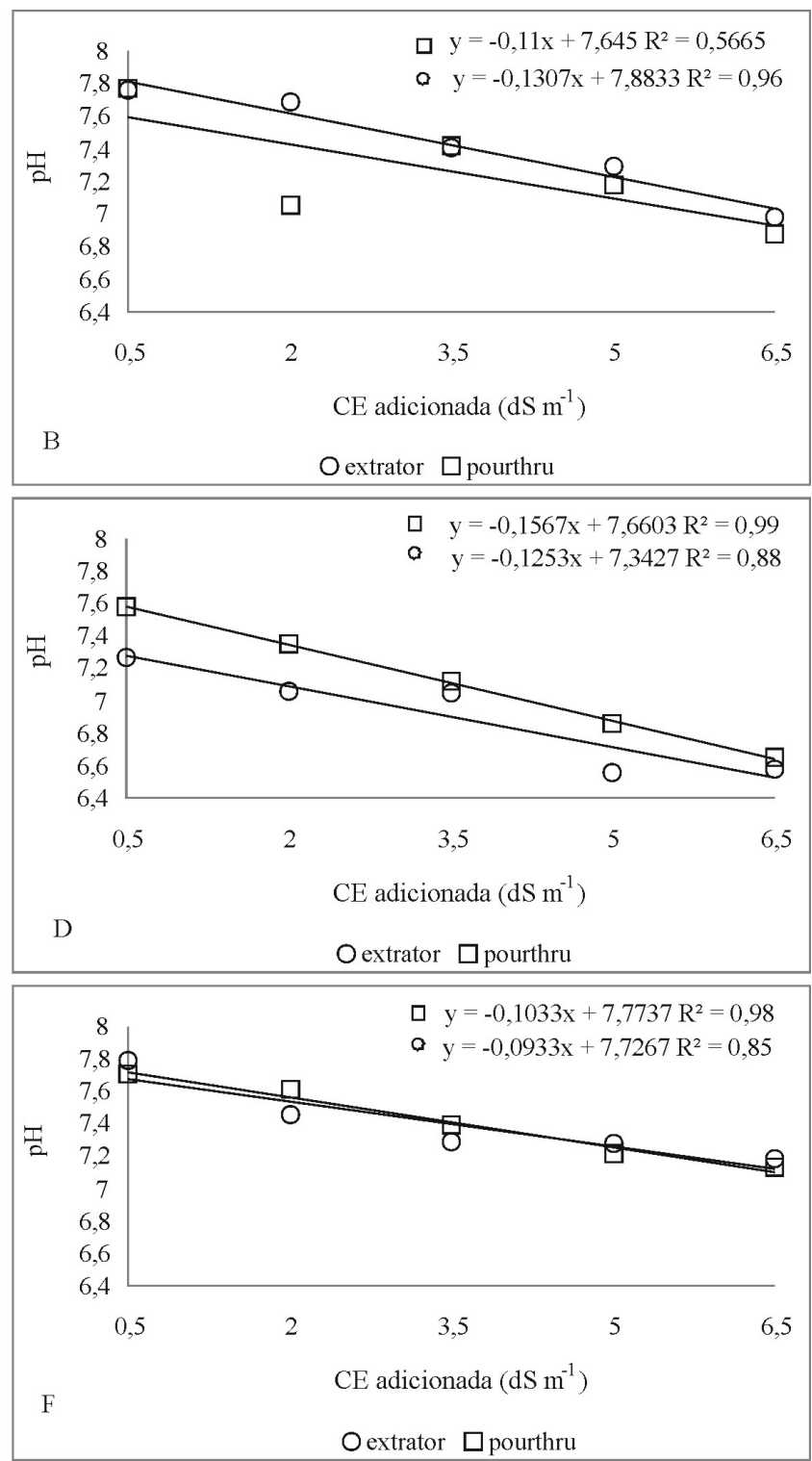

Figura 2. Valores de $\mathrm{pH}$ obtidos pelas metodologias do extrator e pourthru em função das condutividades elétricas adicionadas aos 9 (A), 16 (B), 23 (C), 37 (D), 44 (E), 51 (F) dias após aplicação. Botucatu, UNESP. 2006.

Figure 2. $p H$ values obtained by the methods of solution extractor and pourthru as a function of electrical conductivity added, to 9 (A), $16(B), 23(C), 37(D), 44(E), 51(F)$ days after application. Botucatu, UNESP. 2006.
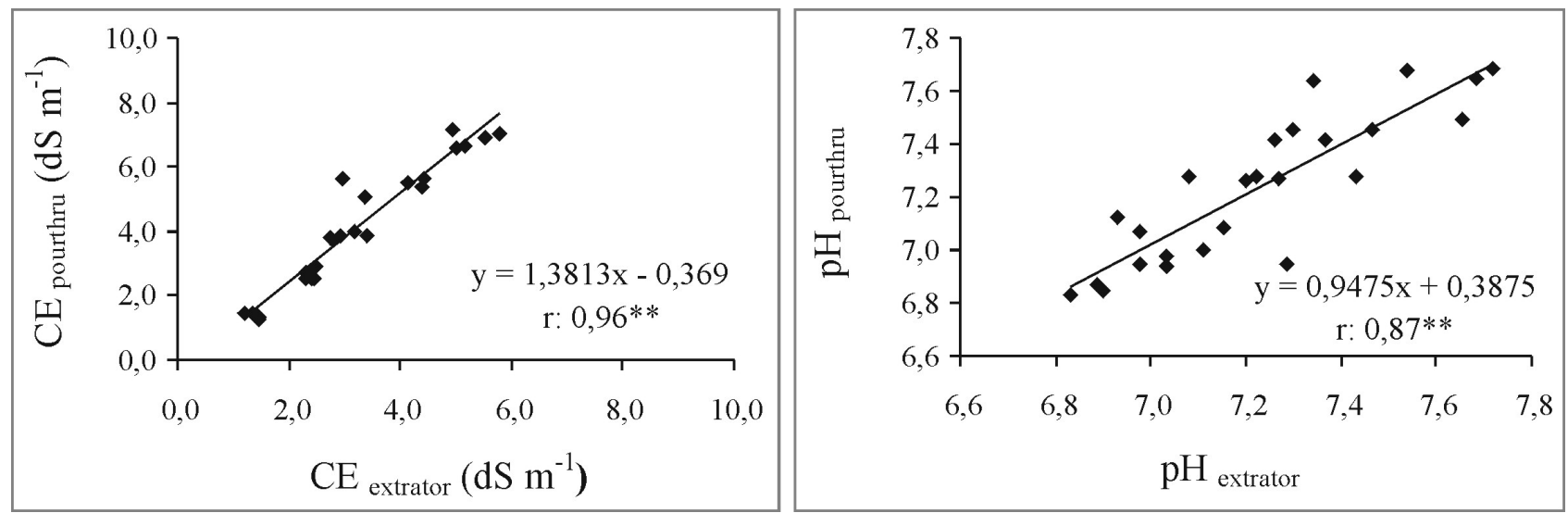

Figura 3. Correlação entre a condutividade elétrica $(\mathrm{CE})$ e valor de pH da solução do substrato obtida pela metodologia do extrator de solução e "pourthru". Botucatu, UNESP. 2006.

Figure 3. Correlation between electrical conductivity (EC) and pH value obtained by the methodology of the solution extractor and pourthru. Botucatu, UNESP. 2006. 